\title{
Relendo o último capítulo de Understanding media. Um tributo a Marshall McLuhan no centenário de seu nascimento
}

\author{
Rereading the final chapter of Understanding Media. A tribute to Marshall McLuhan on the \\ centenary of his birth
}

\begin{abstract}
Doutor em Conservação dos Bens Culturais (Bens Arquivísticos e Livrários) pela Universidade de Parma Especialista em Arquivística, Paleografia e Diplomática pelo Arquivo de Estado de Parma. Docente do Curso de graduação em Bens Artísticos, Teatrais, Cinematográficos e das Novas Mídias da Universidade de Parma. E-mail: $\underline{\text { alberto.salarelli@unipr.it }}$
\end{abstract}

\begin{abstract}
Resumo
O artigo examina os argumentos de Marshall McLuhan no último capítulo de seu livro Understanding Media, relativos ao impacto da tecnologia da informação na organização social. Escritos há cinqüenta anos, esses argumentos revelam, hoje, com a realização da sociedade em rede, seu valor pleno, contribuindo para um verdadeiro "Renascimento McLuhan". Em específico, o ensaio aponta que McLuhan não era absolutamente otimista ou entusiasta em relação à revolução digital, mas sim, pelo contrário, expressava uma profunda preocupação sobre o poder crescente da tecnologia. Essa visão do mundo coloca em relação as reflexões de McLuhan com as teorias de autores contemporâneos tais como Lanier, Carr e Schirrmarcher.
\end{abstract}

Palavras-chave: Marshall McLuhan; Teoria da Media; Sociedade em Rede; Tecnopoder.

\begin{abstract}
The article examines the arguments made by Marshall McLuhan in the last chapter of his book Understanding Media related to the impact of information technology on social organization. Written fifty years ago, they show today, with the advent of the network society, their full value by contributing to a real "McLuhan renaissance". Particularly this essay points out that McLuhan was not at all optimistic and enthusiastic about the computer revolution but instead, he expressed a very strong concern about the growing power of technology.

This worldview relates McLuhan's thought to the theories of contemporary authors such as Lanier, Carr and Schirrmarcher.
\end{abstract}

Keywords: Marshall McLuhan; Media Theory; Network Society; Technopower

I

O centenário do nascimento de Marshall McLuhan (Edmonton, 21 de julho de 1911 Toronto, 31 de dezembro de 1980) acontece em um momento particularmente feliz para a reavaliação de sua obra. Há alguns anos, com efeito, um "renascimento McLuhan", como o definiu Gary Genosko (2005), está, finalmente, libertando a visão do mundo do grande pensador canadense e de toda a escola de Toronto daquele gueto em que boa parte da academia a tinha, apressadamente, relegada sob o rótulo de determinismo tecnológico. Um

InCID: R. Ci. Inf. e Doc., Ribeirão Preto, v. 2, n. 2, p. 3-18, jul./dez. 2011. 
rótulo infamante, para muitos sociólogos das mídias mas, principalmente, injusto em relação às brilhantes intuições mcluhanianas, hoje evidentemente cada vez mais essenciais para entender a realidade da economia de mercado pós moderna, na qual prevalece o valor de troca (num primeiro momento, pois lidamos com uma economia informacional) sobre o valor de uso. Geert Lovink afirma que a erosão da mídia de massa não se percebe tanto pela menor utilização das mídias mainstream (televisão e rádio continuam a manter cotas extremamente significativas nas dietas midiáticas das populações dos países mais avançados e a perda de copias impressas dos jornais cotidianos vê, em compensação, um crescimento de suas consultas em PC, tablet e smartphone), mas pelo fato que "a mensagem impressa ou transmitida via éter perdeu sua aura e as notícias são consumidas como se fossem produtos de entretenimento" (LOVINK, 2008, p. 63). Sem dúvida, uma das causas dessa mudança de postura perante o consumo de informação é representada pelo crescimento da população de Internet, que não somente utiliza documentação produzida pelo outro mas, conforme a lógica participativa que caracteriza os social networks, adiciona continuamente dados, notícias, comentários e conversas, inflacionando, assim, o sistema informativo global a ponto de esvaziá-lo cada vez mais de significado: nunca, na história humana, a informação, graças à máquina informacional por excelência (RAWLINS, 2001, p. 21), o computador, adquirira um valor tamanho a ponto de batizar uma época (a era da informação) e que conta tanto na economia mundial ${ }^{1}$, caracterizando uma cultura global não pelo que ela veicula, mas pela sua própria estrutura.

Como se sabe, a relação entre evolução tecnológica e organização social é uma chave de leitura que pode ser aplicada à toda a história do homem. Comentando o famoso aforismo de McLuhan "o meio é a mensagem”, Neil Postman começa desde a história da invenção do alfabeto por obra de Teuth, contada no Fedro de Platão, para chegar à conclusão, na esteira da lição do mestre de McLuhan, Harold Innis, de que sempre as novas técnicas "alteram a estrutura de nossos interesses: as coisas nas quais pensamos. Elas alteram o caráter de nossos símbolos: as coisas com as quais pensamos. Enfim, alteram a natureza da comunidade: o terreno no qual se desenvolvem os pensamentos" (POSTMAN, 1993, p. 25).

A universalidade desses assuntos e sua validade no tempo não podem, todavia, esconder o fato de que, exatamente hoje, com o trunfo da informática e da telemática, o meio nos aparece mais do que nunca como a verdadeira mensagem. Finalmente descartada a critica

\footnotetext{
${ }^{1}$ Mais de $70 \%$ da economia dos países do G7 se fundamenta em assets intangíveis, isso é, basicamente informacionais, como relata Luciano Floridi (2010, p. 5)
} 
fútil contra McLuhan de não considerar adequadamente o valor do conteúdo da mensagem, uma critica que desconsiderava o estilo dele de escrita - que fazia do paradoxo, a arma fundamental para maravilhar e provocar o leitor e, principalmente os colegas da academia (LOGAN, 2010, p. 353) - assim como desconsiderava a característica fundamental de seu pensamento - que prefere a visão sintética ao percurso analítico (BARILLI, 2006, p. 71-74) podemos, finalmente, reconhecer como o percurso de pesquisa do pensador canadense se revela, hoje, de grande utilidade para entendermos as características e as contradições da época contemporânea. Se é verdade, como escrevia Tolstoj, que "entre as inúmeras subdivisões que podem ser feitas dos fenômenos da vida, uma pode ser a substância e fenômenos nos quais prevalece a forma" (TOLSTOJ, 1990, p. 828), por quê a escolha mcluhaniana de privilegiar o estudo do meio possui, hoje, uma importância determinante?

Isso se deve ao fato da informação ser uma tecnologia leve e simbólica, altamente flexível e, exatamente por isso, possível de ser aplicada, com as características específicas de sua própria linguagem, a uma gama extremamente ampla de situações. É necessário, porém, reconhecer que, se nos limitássemos a essa leitura, a revolução atual não apresentaria diferenças substanciais em relação àquela alfabética. O ponto de ruptura fundamental consiste no fato de que os sistemas informacionais baseados na eletricidade são capazes de controlar os processos das máquinas. Em outras palavras, são, basicamente, sistemas de controle (BENIGER, 1986). McLuhan resgata plenamente esse aspecto duplo que caracteriza a revolução da informação, utilizando uma metáfora eficaz: "although an automated plant is almost like a tree in respect to the continuous intake and output, it is a tree that can change from oak to maple to walnut as required" (McLUHAN, 1994, p. 356).

\section{II}

A relação entre informação e automação é a questão que Marshall McLuhan coloca no centro do último capítulo de sua obra mais famosa, Understanding media. Automação é um termo que - genericamente - indica o uso de aparelhos e sistemas que apresentam um determinado nível de independência operacional no desenvolvimento das funções a eles confiadas. Se esse conceito, em sentido amplo, pode ser aplicado desde o século XIX - isto é, no pleno fulgor da era mecânica - para descrever o funcionamento de aparatos particulares em atividades industriais específicas, como os bem conhecidos como o do tear automático de Joseph-Marie Jacquard e da máquina tabuladora de Herman Hollerith, é somente depois da 
Segunda Guerra Mundial, com a invenção e aperfeiçoamento do computador, que se assiste à possibilidade de aplicar uma gestão automatizada a todo processo industrial, descritível como um modelo informacional, isto é, no qual os dados, as operações e as funções podem ser traduzidas em instruções a serem submetidas ao programador: "automation is information" (p. 346) escreve McLuhan na abertura do capítulo, para sancionar esse binômio, hoje considerado indissolúvel. Por essa razão, ou seja, com uma visão retrospectiva, não é absolutamente secundário contextualizar cronologicamente a afirmação mcluhaniana contida em Understanding media, volume publicado pela primeira vez em 1964: naquela época, os poucos computadores à disposição ainda eram extremamente caros e ocupavam espaços notáveis, alem de precisar de competências técnicas elevadas para poder realizar uma gama de operações que, hoje, nos aparecem irrisoriamente limitadas. Mesmo assim, em filmes como Desk set e The battle of the sexes, ambos do final da década de 1950, emerge, debaixo da patina de gênero de comédia brilhante, uma questão dramática: em ambas as películas, interpretadas por atores celebérrimos entre o grande público, como Spencer Tracy, Katerine Hepburn e Alec Guinness, a rotina cotidiana da gestão empresarial é revirada pelas tirânicas obrigações de um "cérebro eletrônico" imposto pela direção com a finalidade de otimizar a gestão documentária. Os culpados são, em primeiro lugar, os funcionários, considerados como simples "passa-papeis", como relíquias de uma enferrujada burocracia de papeis e arquivos. Somente a moral consoladora do final dos dois filmes salva a sabedoria da tradição fundada nos hábitos e na experiência dos trabalhadores, capazes de remendar as confusões provocadas pelo computador, mas a admoestação dada ao público é clara: estão preparados para enfrentar a revolução informática que logo mudará o mundo?

O traço profético do último capítulo de Understanding media não consiste, assim, em resgatar o enorme porte de uma incomensurável mudança tecnológica, evidentemente já percebida, pelo menos em nível de classe media - a primeira, com efeito, a ser afetada pela concorrência do trabalho desenvolvido pelos processadores - quanto em delinear suas características mais significativas de desenvolvimento futuro. De fato, McLuahn conhecia de dentro, o potencial da indústria informática e, principalmente, mais que as inovações propostas na vertente tecnológica, era bem consciente dos objetivos do mercado aos quais ela podia aspirar. Nos anos em que vinha à luz Understanding media McLuhan, como se sabe, desenvolvia consultorias para a IBM sobre um tema que é um verdadeiro programa: "Vocês não devem mais construir máquinas de escrever, mas oferecer ao cliente a resposta às perspectivas de desenvolvimento de suas atividades"(GAMALERI, 2006, p. 30). 
Em outros termos, não deve ser atribuída a McLuhan uma argúcia particular por destacar um tema atual mas, sim, por ter oferecido uma primeira visão na íntegra dessa revolução tão avassaladora que revolveu por inteiro nossas existências: como mostra o subtítulo do capítulo, learning a living, daí em diante torna-se necessário estar prontos a morar em um mundo novo, a projetar uma vida nova em um horizonte tecnológico radicalmente transformado.

III

Quais são os traços que dão qualidade a essa transformação de porte incomensurável? Relendo, depois de quase meio século, o último capítulo de Understanding media, isso é, adotando um olhar retrospectivo que permite abarcar quase por inteiro o desenvolvimento da história da informática, pode-se apreciar a extraordinária capacidade de McLuhan em saber "antecipar os jogos". Com efeito, se o resto de seu volume, capítulo após capítulo, é uma análise ex-post dos efeitos sociais das diversas mídias em análise, em suas páginas conclusivas a reflexão se concentra em uma tecnologia na época ainda balbuciante, ainda in cuna, ou seja, ainda no berço, para usar uma expressão querida aos historiadores do livro para apontar a prática artesanal dos prototipógrafos da segunda metade do século XV e, ao mesmo tempo, também os primeiros livros impressos: os incunábulos. É isso: se para McLuhan era, sem dúvida, mais confortável estabelecer uma relação entre a invenção guteberguiana e as características das modernas formas de Estado, já que aquele processo histórico concluira-se, de fato, havia tempo, bem mais complexo era imaginar como nos envolveria aquela "criança" sem beleza e grosseiro - o computador - que, mesmo assim, já na época concentrava sobre si grandes expectativas, não isentas de preocupações de igual tamanho. Não é por acaso que, entre as muitas incógnitas da época, uma das poucas certezas era o efeito de compressão perceptível das categorias kantianas de espaço e de tempo. Essa certeza se revelava pela sua fonte de alimentação, a eletricidade, e pelos efeitos que a mesma já manifestara nas tecnologias da comunicação, às quais fora aplicada desde o século XIX. Portanto, em virtude dessa trajetória já percorrida, McLuhan se pergunta, retoricamente, "it is strange then, that electricity should confer on all previous human organization a completely new character?” (p. 351-352). 
Menos óbvia, todavia, era a possibilidade de intuir quais formas tomaria a aplicação da eletricidade à capacidade de representação simbólica dos computadores e de sua virtualmente ilimitada flexibilidade, Considere-se a seguinte afirmação:

\footnotetext{
It is a pricipal aspect of the electric age that it establishes a global network that has much of the character of our central nervous system. Our central nervous system is not merely an electric network, but it constitutes a single unified Field of experience (McLUHAN, 1994, p. 348)
}

No final das contas, é fácil, para nós, hoje, compreender esse conceito, graças à cotidiana familiaridade que temos com as redes digitais e com o hábito, já arraigado, de aplicar o paradigma de Internet, deslocando-o do plano infraestrutural para aquele da organização dos conteúdos, chegando às mais adiantadas formas de governance empresarial. Como não enxergar, por exemplo, no modelo de gestão das informações proposto pela Wikipédia, uma declinação concreta daquele campo unificado de experiência do qual fala McLuhan? Também na década de 1960, claro, os sistemas de comunicação broadcast, como rádio e televisão, podiam sugerir a idéia de uma participação experiencial comum por parte daquele sujeito coletivo chamado "público". E não há dúvidas de que essa idéia pesou, de maneira determinante, na formulação do conceito de "aldeia global". Vale a pena, porém, relembrar que, mesmo no caso de acontecimentos de porte global, como a conquista do espaço ou a guerra do Vietnam, tratava-se de experiências geograficamente segmentadas, em função da bacia de cobertura do sinal e, fundamentalmente, induzidas por uma fruição passiva do espectador. McLuahn, pelo contrario, sugere uma perspectiva diferente, que busca ir alem da videoesfera televisiva: a imagem que ele propõe é a de uma rede que cobre o globo inteiro e que, na comparação com o sistema nervoso humano, adquire os caracteres de um complexo orgânico, no qual as informações se deslocam em uma modalidade bidimensional. Não há dúvida alguma de que a visão do estudioso canadense seja devedora das hipóteses formuladas por Norbert Wiener, no que diz respeito ao conceito de organicidade sistêmica fundada nos princípios de descentralização e de interatividade, e de Vannevar Bush, pelo modelo hipertextual de gestão das informações. A esses elementos McLuhan adiciona mais um, que consiste em considerar a rede elétrica global orgânica e descentralizada como o lugar onde se realiza uma atividade incessante de intercâmbio de informações, onde o "consumer becomes producer" (McLUHAN, 1994, p. 349) ou, como diríamos hoje com um neologismo na moda, prosumer. A eletricidade oferece proeminência ao processo, escreve McLuhan, e isso significa que ela, como, por outro lado, todas as outras mídias, não são uma "coisa" mas uma "função" (BARILLI, 2006, p. 73) e que, consequentemente, o aspecto mais singular dessa 
rede, que envolve o planeta inteiro, é o seu pulsar de vida, sincrônico com nossos sistemas nervosos, dos quais representa a mais realizada extensão.

Dito várias vezes, inclusive por Armand Mattelart (2002, p. 66), que essa visão vital ou, talvez mais propriamente, funcionalista, de McLuahn, se deve à influência exercida sobre ele por Pierre Teilhard de Chardin, autor que, por outro lado, é expressamente citado tanto em Understandig media como em The Gutemberg galaxy. Com efeito, como sugeriram, entre outros, James Curtis (1978, p. 83) e Elena Lamberti (2000, p. 133), é necessário ampliar em muito as referencias culturais que constituem o pano de fundo para essa forma de entender as mídias como um sistema orgânico até chegar, por admissão direta do próprio McLuhan, em São Tomás de Aquino, o primeiro que lhe sugeriu o princípio de complementaridade entre todas as formas criadas (LAMBERTI, 2000, p. 137). Esse numero mais amplo de referencias culturais permite desprender o pensamento de McLuhan de uma dependência absoluta e exclusiva em relação à lição de Teilhard de Chardin, operação que oferece a possibilidade de pontuar a originalidade de sua reflexão em relação a duas questões fundamentais.

A primeira diz respeito ao papel de cada indivíduo em relação ao sistema integrado das mídias eletrônicas. Conforme Teilhard de Chardin, o indivíduo está fadado a se aniquilar em uma inteligência coletiva superior, em um "sentido cósmico" no qual "os homens param de representar individualidades fechadas em si, para se tornar partes de um Todo"(TEILHARD DE CHARDIN, 1997, p. 168), um ponto de chegada que, para McLuhan, não é certamente tão óbvio. Com efeito, se é verdade que, em relação à exaltação do pensamento individual, uma das características fundamentais trazidas pela invenção gutemberguiana da imprensa, as mídias eletrônicas tendem a achatar a fragmentação entre os saberes e a solicitar a integração social, não há razão para que esse processo aconteça de forma linear, ou que uma nova geração de mídia se imponha sobre as anteriores. Assim não se pode afirmar que, a dimensão dialética com que cada indivíduo mantém com as ferramentas que o cercam, potencializando seus efeitos mas, também, interpretando suas potencialidades, deva ser considerada irrelevante em relação à união mística na "noosfera" de Chardin. Pelo contrario, conforme McLuhan, a chegada da era eletrônica - e o novo paradigma geral que ela impõe de maneira traumática à sociedade - obriga à necessidade de individualizar formas adequadas de aprendizagem que sejam capazes de tutelar a dignidade e a personalidade do indivíduo. 
O último capítulo de Understanding media é constantemente permeado por essa pedagogia pertinente aos novos tempos, que seja capaz de superar as divisões tradicionais entre os diversos campos do saber, a partir do momento em que "continued in their present patterns of fragmented unrelation, our school curricula will insure a citizenry unable to understand the cybernated world in which they live" (McLUHAN, 1994, p. 347). Trata-se, como se pode observar, de um problema até hoje em aberto: em um brilhante ensaio, há poucos anos atrás, dedicado aos temas da globalização, Thomas Friedman insistiu sobre o papel estratégico ocupado pelas estruturas educacionais do mundo avançado. Por exemplo, referindo-se à instrução universitária, ele escreve que essa

\footnotetext{
Adquire uma importância cada vez maior conforme o mundo se achata, pois o desenvolvimento tecnológico eliminará os velhos trabalhos e criará novos e mais complexos com uma velocidade muito maior em comparação com o que aconteceu na fase de passagem da economia agrária para aquela industrial.
}

A questão, todavia, diz respeito à educação desde o primeiro grau até a pós graduação, já que o lifelong learning é um dos mantras mais presentes quando se fala em sociedade do conhecimento. Com efeito, se a intuição mcluhaniana de que a aprendizagem se tornaria, em poucos anos, a "tipologia principal de produção e de consumo" (McLUHAN, 1994, p. 374), sendo hoje considerada um ponto central de todas as economias avançadas, menor compromisso foi dedicado - como observa várias vezes Friedman - à estruturação de percursos de aprendizagem transversais e inovadores, capazes de interpretar a complexidade do mundo contemporâneo. Era essa, todavia, a missão da qual McLuhan se encarregou, como teve ocasião de declarar ele mesmo em 1967 em uma entrevista confiada a Richard Kostelanetz do "New York Time Magazine": questionado sobre o sentido de sua pesquisa, o estudioso respondeu que:

my entire concern is to overcome the determinism that results from the determination of people to ignore what is going on. Far from regarding technological change as inevitable, I insist that if we understand its components we can turn it off any time we choose (KOSTELANETZ, 1967).

E, é preciso lembrar, a exigência de uma nova pedagogia de matriz socrática, na qual o professor, com seus próprios conhecimentos, sai da posição do professor que tudo sabe para formular perguntas instigantes aos alunos, método privilegiado que McLuhan identificava para essa reprogramação dos percursos escolares (MAFFIA, 2006, p. 287). Um método que, como denunciava Martha Nussbaum, é hoje cada vez mais ignorado e não reconhecido, com riscos graves para a manutenção de uma democracia vital, respeitosa e responsável (NUSSBAUM, 2010, cap. 4). Como não reconhecer, nessa uma nova pedagogia proposta por 
McLuhan, a necessidade fundamental de proteger a personalidade individual contra um uso acrítico dos instrumentos da comunicação? Como assimilar essa posição com a de Teilhard de Chardin em que as inteligências individuais se fundirão em uma mente superior?

Com o senso critico com que cada um de nós vive a contemporaneidade, e olhando o futuro com otimismo, coloca-se o segundo elemento de divergência profunda entre o jesuíta e o professor. Um sociólogo italiano, Paolo Jedlowski, recentemente escreveu:

\begin{abstract}
A experiência de uma excesso de tarefas, atividades, encontros, possibilitada pela velocidade com a qual nos deslocamos e comunicamos é, sem dúvida, difundida [...], mas tal excesso corresponde também, necessariamente, a uma reduzida capacidade de refletir sobre as motivações e as finalidades pelos quais nos deixamos envolver (JEDLOWSKI, 2005, p. 63).
\end{abstract}

O efeito nocivo da combinação produzida pela velocidade e pela quantidade de informações veiculadas pela mídia, há tempo é conhecido, mesmo que não fosse identificado com o nome que hoje, comumente, lhe é atribuído: information overload. Por exemplo, Georg Simmel, já nos primeiros anos da século XX, percebeu o desconforto provocado pelo excesso de informação para o morador da grande cidade (SIMMEL, 1998, p. 553). Ainda, em 1956, George Miller, um dos grandes nomes da psicologia mundial, identificava os traços significativos de uma verdadeira síndrome de bloqueio de dados no cérebro humano: "confusion will appear near the point that we are calling his «channel capacity»" (MILLER, 1956, p. 83). Com a chegada dos sistemas informáticos e telemáticos essa tendência amplifica-se desmedidamente: a sociedade que adquire forma através de uma comunicação principalmente fundamentada em sistemas reticulados tende a constituir um sujeito conectivo comparável a um imenso formigueiro (LONGO, 2003, p. 23). Assim, poderíamos realmente estar perante a perspectiva escatológica que Dostoevskij descreveu, dando voz ao Grande Inquisidor, afirmando que a máxima aspiração do homem, aquilo que busca nesse mundo, é, "na frente de quem se ajoelhar, a quem confiar sua consciência e de que maneira, finalmente, reunir todos em um formigueiro comum, enquanto a existência de uma união universal é a terceira e última angústia dos homens" (DOSTOEVSKIJ, 2005, p. 343). Em outras palavras, o sucesso extraordinário das ferramentas de gerenciamento da informação de estrutura reticular está se impondo como a única macrovisão plausível do mundo, que tudo inclui e nada exclui: a network society (CASTELLS, 2002, p. 13-14). Inevitável, portanto, que sejam muitos aqueles dispostos a se ajoelhar perante a divindade da Rede, mesmo ao preço de se aniquilar nela, como o Narciso do mito, reinterpretado em chave mclhuaniana. A posição otimista dos discípulos ideais de Teilhard de Chardin, entre os quais podemos listar Nicholas Negroponte e 
Pierre Levy, é a posição de quem, na contenda entre a primazia da comunicação (que privilegia a dimensão coletiva e o significante) e aquela do conhecimento (que se fundamenta na reflexão pessoal e no significado), privilegia a primeira em detrimento da segunda, frequentemente sem considerar que, se é verdade que os dois planos do discurso sempre se confrontaram, é todavia evidente como a hipertrofia do meio está levando à progressiva aniquilação do sentido da mensagem. Ora, é peculiar perceber como McLuhan foi, até sua morte, praticamente ignorado pelos grandes exegetas das formas de comunicação e organização social, tais quais Michel Foucault, Jacques Derrida, Jacques Lacan, Gilles Deleuze, Jürgen Habermas, Louis Althusser e Jean François Lyotard (POSTER, 2010, p. 2) e, em seguida, colocado no time dos otimistas, com base no fato que seu interesse para com os instrumentos da comunicação impedira-lhe de compreender o quadro na íntegra, político e social, no qual esses desenvolviam suas potencialidades ${ }^{2}$. Somente mais recentemente, graças aos estudos de autores como Marchand e Genosko, fez-se justiça à posição real de McLuhan em relação ao destino da humanidade perante as novas tecnologias, uma posição que deve ser avaliada com cuidado em sua complexidade, onde coexistem a curiosidade do cientista que observa um mundo em repentina evolução: "to be born in this age is a precious gift"; a esperança do católico na capacidade do homem de crescer e aprender: "I have a great faith in the resiliency and adaptability of man"; e o desaconchego existencial pessoal perante as mudanças sociais e psíquicas provocadas pelas novas mídias:

I do see the prospect of a rich and creative retribalized society - free of the fragmentation and alienation of the mechanical age - emerging from this traumatic period of culture clash; but I have nothing but distaste for the process of change. As a man molded within the literate Western tradition, I do not personally cheer the dissolution of that tradition through the electric involvement of all the senses: I don't enjoy the destruction of neighborhoods by high-rises or revel in the pain of identity quest. No one could be less enthusiastic about these radical changes than myself. I am not, by temperament or conviction, a revolutionary; I would prefer a stable, changeless environment of modest services and human scale. TV and all the electric media are unraveling the entire fabric of our society, and as a man who is forced by circumstances to live within that society, I do not take delight in its disintegration.

Teríamos nos poupado de muitas interpretações ruins sobre o pensamento de McLuhan se seus detratores nas várias épocas tivessem lido a entrevista que o estudioso concedeu ao periódico "Playboy" em março de 1969 (NORDEN, 1969)³. Naquelas páginas,

\footnotetext{
${ }^{2}$ Uma excelente reconstituição do debate critico suscitado no mundo acadêmico com o aparecimento dos trabalhos de McLuhan encontra-se em Baran e Davis, 2008, cap. 8.

3 Vale a pena lembrar como em seu período áureo - isso é, até o limiar do século XXI - "Playboy" publicava todo mês uma entrevista extremamente ampla e aprofundada com celebridades da época. A escolha de McLuhan de aparecer nesse periódico faz parte de uma estratégia de comunicação completa que não deixava de utilizar
} 
das quais foram retiradas as citações acima, McLuhan apresenta, como nunca em outras ocasiões, uma síntese muito clara de sua postura de pesquisador curioso e atento às mudanças e, ao mesmo tempo, de homem profundamente desconcertado pelos efeitos das mesmas. Assim, rotulá-lo como "otimista" pode ser prático, porém, com efeito, revela-se uma forma superficial e redutiva de entender o aporte integral de seu pensamento. Não é por acaso que, hoje, os estudiosos que se apelam com maior convicção ao trabalho dele são inseridos nos manuais de teoria das mídias no campo oposto, entre os pessimistas (ver, por exemplo, BOURDON, 2001, p. 32). Nesse caso, também, trata-se de uma operação excessivamente simplificadora, ainda que compreensível pela clareza da exposição. Essa operação, todavia, tem o mérito de enfocar uma questão importante: nos dias de hoje, quem interpreta a sociedade contemporânea realçando o papel proeminente que as tecnologias têm sobre ela, tende a oferecer uma visão critica, no lugar de oferecer um quadro do melhor dos mundos possíveis. Em alguns casos, perante o curriculum respeitado dos autores - claramente não rotuláveis como tecnofóbicos "de carteirinha" - essas criticas relativas aos efeitos sociais e, até, antropológicos, produzidos pela técnica, tiveram um sucesso editorial de ressonância internacional notável. Os ensaios aos quais nos referimos nao You are not a gadget, de Jaron Lanier, The shallows: what the Internet is doing to our brains, de Nicholas Carr e Playback, de Frank Schirrmacher (LANIER, 2010; CARR, 2010; SCHIRRMACHER, 2009).

A tese de base de Lanier, um informático ao qual se devem estudos fundamentais com relação às interfaces homem/máquina, consiste na afirmação de que as tecnologias digitais nos colocam em uma condição de lock in face a seus próprios resultados. Em outras palavras, algumas escolhas de base feitas no passado, relativamente ao design dos computadores e dos aplicativos utilizados por todos nós hoje em dia, nos trancafiaram dentro de uma lógica de gestão das informações que fugiram ao controle de seus próprios projetadores, impondo-nos escolhas obrigatórias, geradas dentro da estrutura dos sistemas de programação. Esse fenômeno, que se encontra em todos os níveis, desde a organização dos ícones em nossa tela, até o modus operandi colaborativo das redes sociais, produz êxitos desastrosos, como a asfixia de qualquer cenário alternativo na organização dos dados vinculada pelas rígidas e maniqueístas alternativas do dígito binário ou, ainda, como pode ser observado face todas as aplicações 2.0, a convicção disseminada de que as multidões interconectadas e falantes podem representar um degrau de inteligência superior em relação à dos indivíduos singulares.

qualquer meio que lhe permitisse o contato com um público mais amplo em relação ao circuito acadêmico. Eric Norden era um dos entrevistadores mais importantes da revista: com efeito, alem da entrevista dedicada a McLuhan, realizou aquelas de Truman Capote, Stanley Kubrick, Gore Vidal e Stephen King. 
Nicholas Carr (outro tecnófilo da primeira leva, autor de editoriais de revistas prestigiosas e, por longo tempo, diretor da "Harvard Business Review"), como se deduz do titulo de seu bestseller, coloca-se de maneira até mais avançada: o uso das novas tecnologias está modificando profundamente a atividade de nosso cérebro, na medida em que as áreas ativadas pela prática da leitura realizada através do livro impresso são subutilizadas, enquanto aquelas relacionadas à leitura na tela tendem à hipertrofia. O resultado, inevitável, é que o pensamento lógico-dedutivo, o aprofundamento interior, o exercício da faculdade da memória, isso é, as habilidades específicas relacionadas à cultura das páginas impressas, estão fadadas a se tornarem secundárias em relação às competências fisiológicas necessárias para a fruição das novas mídias, que privilegiam a paratáxis - isso é, o multitasking - mais que a hipotáxis.

Trata-se de uma reflexão compartilhada também por Frank Schirrmacher que, em seu trabalho, sem se exceder na provocação, afirma que, em breve, não será mais possível entender "onde começa o computador e onde acaba o cérebro" (cap. 18), prefigurando uma espécie de isomorfismo entre a psique humana e os sistemas de gestão da informação, provocado pelo efeito desses últimos sobre o conjunto de nossas faculdades cognitivas.

Em outras palavras, o que se conclui da leitura dos ensaios desses autores, é a sensação de não termos mais a capacidade de executar um controle humano eficaz sobre aquele sistema de controle automatizado que as máquinas informáticas permitem realizar.

Sem chegar - pelo menos, até agora - às formas dramáticas de rebelião aberta contra o homem por parte dos computadores (como, por exemplo, prefiguraram Asimov e Kubrick), nos encontramos vivendo em uma condição de dependência em relação a eles. Com efeito, as redes telemáticas se apresentam como uma infraestrutura conectiva que torna possível um diálogo direto entre as máquinas, sem mais necessariamente prever a instância de uma intermediação humana: os sistemas informáticos são, cada vez mais, capazes de se autoalimentar. Como conseqüência, os computadores acabam tomando decisões na base de dados transmitidos por outros computadores, em uma espécie de "efeito dominó", com resultados freqüentemente imprevisíveis.

Relendo o último capítulo de Understanding media torna-se evidente como McLuhan foi consciente desses riscos: se o multitasking é um elemento que caracteriza os sistemas de informação - "this is why those involved in automation insist that it is a way of thinking, as much as it is a way of doing. Instant synchronization of numerous operations has ended the 
old mechanical pattern of setting up operations in lineal sequence" (McLUHAN, 1994, p. 349) - isso se deve ao fato deles terem sido criados e desenvolvidos buscando imitar a fisiologia humana: em primeiro lugar, de forma aproximativa, em seguida de maneira cada vez mais sofisticada, pode se fazer com que os computadores simulem "the process of consciousness, just as our electric global networks now begin to simulate the condition of our central nervous system" (p. 351). Essa libertação das atividades rotineiras que os chamados "servomechanisms"desempenharão no nosso lugar, todavia, impõe inevitavelmente um preço, que consiste em testar com firmeza "our inner resources of self-employment and imaginative partecipation in society” (p. 358). Estamos, assim, participando de uma competição na qual está em jogo a sobrevivência daquela categoria do "singular" que Kierkegaard via como único baluarte contra a "confusão do panteísmo" (KIERKEGAARD, 1960, p. 467), um risco, evidentemente, não percebido pelos que não perdem a ocasião para enaltecer as potencialidades dos social networks que, exatamente enquanto sociais, apresentam a desvantagem de limitar a infordiversidade, privilegiando um mínimo comum denominador que é a mídia - algoritmicamente gerada - de recíprocos "rating", "liking" e "linking" disseminados pelos usuários nas várias plataformas 2.0 (METITIERI, 2009).

O que é impactante é como os tecnoentusiastas confundiram essa possibilidade de operar simultaneamente e organicamente em vários níveis, uma possibilidade oferecida, em primeiro lugar, pela convergência de todos os sistemas informacionais rumo ao digital, com a superação definitiva dos vínculos que, no mundo analógico caracterizavam, com suas especificidades, cada meio de comunicação: "the medium is not the message in the digital world" (NEGROPONTE, 1995, p. 71), afirmou Nicholas Negroponte, sem se aperceber de como, pelo contrario, nos dias de hoje, na era da automação, temos a possibilidade de observar, a elevação potencial da técnica, portanto do meio sobre a mensagem. Como observa o filósofo Emanuele Severino:

\footnotetext{
na memória e na comunicação total informático-telemática, a mensagem essencial da técnica é de fato a própria técnica, portanto sua capacidade de organizar as mensagens da memória e da comunicação total. Ou seja, a mensagem autêntica é constituída pelo que, comumente, é considerado como um simples meio, como um medium que serve à transmissão das mensagens. Além da consciência que Marshall McLuhan pode ter tido sobre isso, é esse o sentido mais profundo da afirmação de que o meio é a mensagem (SEVERINO, 1996).
}

Entende-se bem, assim, o pesar que McLuhan experimentava, no decorrer de sua existência, em ver fugir de suas mãos a possibilidade de assistir, em primeira pessoa, à materialização dessa sua fundamental intuição. 


\section{Referências}

BARAN, S. J.; DAVIS, D. K. Mass communication theory: foundations, ferment, and future. Boston, MA.: Wadsworth, 2008.

BARILLI, R. McLuhan o del pensiero sintetico. In: GAMALERI, G. (Ed.) Understanding McLuhan: l'uomo del villaggio globale. Roma: Kappa, 2006. p. 71-79.

BENIGER, J. R. The control revolution: technological and economic origins of the information society. Cambridge, Mass.: Harvard University Press, 1986.

BOURDON, J. Introduzione ai media. Bologna: Il Mulino, 2001.

CARR, N. G. The shallows: what the Internet is doing to our brains. New York: W.W. Norton, 2010.

CASTELLS, M. Galassia internet. Milano: Feltrinelli, 2002.

CURTIS, J. Culture as polyphony: an essay on the nature of paradigms. Columbia: University of Missouri Press, 1978.

DOSTOEVSKIJ, F. I fratelli Karamazov. Torino: Einaudi, 2005.

FLORIDI, L. Information: a very short introduction. Oxford; New York: Oxford University Press, 2010.

FRIEDMAN, T. L. Il mondo è piatto: breve storia del ventunesimo secolo. Milano: Mondadori, 2006.

GAMALERI, G. Knowing McLuhan - Conoscere McLuhan. In: GAMALERI, G. (Ed.) Understanding McLuhan: l'uomo del villaggio globale. Roma: Kappa, 2006, p. 13-47.

GENOSKO, G. McLuhan and Baudrillard: the masters of implosion. London; New York: Routledge, 1999.

(Ed.). Marshall McLuhan: critical evaluations in cultural theory. London; New York: Routledge, 2005.

JEDLOWSKI, P. Un giorno dopo l'altro: la vita quotidiana fra esperienza e routine. Bologna: Il Mulino, 2005.

KIERKEGAARD, S. Gli scritti su se stesso. In: LOWITH, K. (Ed.). La sinistra hegeliana. Bari: Laterza, 1960, p. 460-468.

KOSTELANETZ, R. Understanding McLuhan (In Part), New York Times Magazine, January 29, 1967. Disponível em: <http://www.nytimes.com/books/97/11/02/home/mcluhanmagazine.html>. Acesso em: 14 set. 2011.

LAMBERTI, E. Marshall McLuhan tra letteratura, arte e media. Milano: Bruno Mondadori, 2000. 
LANIER, J. You are not a gadget: a manifesto. New York: Alfred A. Knopf, 2010.

LOGAN, R. K. Understanding new media: extending Marshall McLuhan. New York: Peter Lang, 2010.

LONGO, G. O. Il nuovo Golem. Come il computer cambia la nostra cultura. Roma-Bari: Laterza, 2003.

LOVINK, G. Zero comments: teoria critica di Internet. Milano: Bruno Mondadori, 2008.

MAFFIA, E. Il privilegio di vivere oggi. Intervista di Empedocle Maffia a Marshall McLuhan. In: GAMALERI, G. (Ed.) Understanding McLuhan. L'uomo del villaggio globale. Roma: Kappa, 2006, p. 270-288.

MARCHAND, P. Marshall McLuhan: the medium and the messenger: a biography. Cambridge, Mass.: MIT Press, 1998.

MATTELART, A. Storia della societa dell'informazione. Torino: Einaudi, 2002.

McLUHAN, M. Understanding media: the extensions of man. London; New York: Routledge, 1994.

METITIERI, F. Il grande inganno del web 2.0. Roma; Bari: Laterza, 2009.

MILLER, G. A. The magical number seven, plus or minus two: some limits on our capacity for processing information. Psychological Review, v. 63, p. 81-97, 1956.

NEGROPONTE, N. Being digital. New York: Knopf, 1995.

NORDEN, E. The playboy interview: Marshall McLuhan. Playboy Magazine, Mar. 1969. Disponível em: <http://www.mcluhanmedia.com/m_mcl_inter_pb_01.html>.Acesso em: 14 set. 2011.

NUSSBAUM, M. Not for profit: why democracy needs the humanities. Princeton, N.J.: Princeton University Press, 2010.

POSTER, M. Mcluhan and the cultural theory of media. Media Tropes, v. 2, n. 2, p. 1-18, 2010. Disponível em:

<http://www.mediatropes.com/index.php/Mediatropes/article/viewFile/11931/8817>. Acesso em: 14 set. 2011.

POSTMAN, N. Technopoly: la resa della cultura alla tecnologia. Torino: Bollati Boringhieri, 1993.

RAWLINS, G. Schiavi del computer? Roma-Bari: Laterza, 2001.

SEVERINO, E. La tecnica ormai domina l'inventario globale della conoscenza umana.

Telèma, 5, 1996. Disponível em:

<http://web.archive.org/web/20001011193234/http://www.fub.it/telema/TELEMA5/Severin5.

html>. Acesso em: 14 set. 2011. 
SCHIRRMACHER, F. Payback: warum wir im Informationszeitalter gezwungen sind, zu tun, was wir nicht tun wollen, und wie wir die Kontrolle über unser Denken zurückgewinnen. München: München Blessing, 2009.

SIMMEL, G. Sociologia. Torino: Edizioni di Comunità, 1998.

TEILHARD DE CHARDIN, P. L'energia humana: tra scienza e fede. Parma, Nuove Pratiche, 1997.

TOLSTOJ, L. Guerra e pace. Torino: Einaudi, 1990.

Tradução: Giulia Crippa

Revisão: Solange Puntel Mostafa 